\title{
Neurology
}

Issue: Volume 52(8), 12 May 1999, pp 1689-1691

Copyright: (C) 1999 American Academy of Neurology

Publication Type: [Brief Communications]

DOI: 10.1212/WNL.52.8.1689

ISSN: 0028-3878

Accession: 00006114-199905120-00033

[Brief Communications]

\section{Assessment of cognition in advanced AD: The test for severe impairment}

Jacobs, D. M. PhD; Albert, S. M. PhD; Sano, M. PhD; del Castillo-Castaneda, C. BA; Paik, M. C. PhD; Marder, K. MD; Bell, K. MD; Brandt, J. PhD; Albert, M. S. PhD; Stern, Y. PhD

\section{Author Information}

From the Departments of Neurology (Drs. Jacobs, S.M. Albert, Sano, Marder, Bell, and Stern, and C. del Castillo-Castaneda), Psychiatry (Dr. Stern), and the Gertrude H. Sergievsky Center (Drs. Jacobs, S.M. Albert, Sano, Marder, Bell, and Stern, and C. del Castillo-Castaneda), Columbia University College of Physicians and Surgeons, New York, NY; the Department of Biostatistics (Dr. Paik), Columbia University School of Public Heath, New York, NY; the Department of Psychiatry and Behavioral Sciences (Dr. Brandt), Johns Hopkins University, Baltimore, MD; and the Departments of Psychiatry and Neurology (Dr. M.S. Albert), Massachusetts General Hospital, Harvard Medical School, Boston, MA. Supported by federal grants AG07370, AG08702, and RR00645, and the Taub Center for Alzheimer's Disease Research. Received October 30, 1998. Accepted in final form February 10, 1999.

Address correspondence to Dr. Diane Jacobs, Gertrude H. Sergievsky Center, 630 West 168th Street, New York, NY 10032; e-mail: dj32@columbia.edu

\section{Article abstract}

The Test for Severe Impairment (TSI) was compared with the Mini-Mental State Examination (MMSE) and a modified MMSE (mMMSE) in a multisite, longitudinal study of AD. The TSI correlated highly with the MMSE $(r=0.83)$ and the mMMSE $(r=0.82)$, but was not redundant. There was a wide range of scores on the TSI among those scoring in the severely impaired range on the MMSE and mMMSE. The slope of cognitive change over time detected by the TSI was greater than that revealed by the MMSE or the mMMSE. Performance on the TSI was a significant predictor of survival. The TSI is a valid measure that is sensitive to cognitive change over time in severely demented patients with AD.

Traditional neuropsychological tests and mental status examinations have limited utility in severely demented patients due to floor effects (i.e., patients fail all, or nearly all, items). To assess cognitive functioning better in these patients, several instruments have been developed.1-3 We incorporated the Test for Severe Impairment (TSI)1 into a longitudinal study of AD. We examined change on the TSI over time and compared subjects' performance on the TSI with the Mini-Mental State Examination (MMSE) and a modified version of the MMSE (mMMSE).

Method. Participants. Participants were drawn from a longitudinal multicenter study of AD. Study design and cohort characteristics have been described previously. 4

Procedures. For all participants, cognition was assessed at 6-month intervals with the mMMSE.5 Participants at the Johns Hopkins site also received the standard 30-item MMSE. 6 The TSI was introduced in year 4 of data collection. The 24-item TSI was developed to measure cognition in persons with advanced dementia, and has been validated in dementia patients who scored less than 10 points on the MMSE.1 All three instruments were administered by a research assistant using standard procedures.

Preliminary examination of TSI and mMMSE data in this study revealed little variability in the TSI score among participants scoring 30 points or higher on the mMMSE (corresponding to approximately 16 points on the MMSE). Almost all of these patients obtained the maximum score on the TSI. As a result, a procedural change was implemented in which the TSI was no longer administered to participants scoring 30 points or higher on the mMMSE. Therefore, data included in the analyses of longitudinal change were restricted to visits at which subjects scored less than 30 points on the mMMSE. 
Data analysis. Raw scores were converted to percent-correct scores (i.e., [score x maximum score] x 100) to account for differences in the range of possible scores on the tests. The cross-sectional association between the TSI and the mMMSE was determined by examining scatter plots of the two variables from the first visit at which a TSI was available, and by computing Pearson's product moment correlation coefficients. To assess longitudinal decline, the slope of change in test score over time was calculated for each subject who had three or more visits (requisite for computing slope). The difference between longitudinal slopes on the mMMSE and the TSI was assessed using paired $t$-tests. Cox regression analysis was used to compare initial TSI and mMMSE scores as predictors of survival until death. All analyses were then repeated on the subset of participants from Johns Hopkins who also had received the standard MMSE to compare the TSI and the MMSE.

Results. TSI and mMMSE. The TSI and mMMSE were administered to 125 participants during a total of 474 visits. At the time of the initial TSI, these AD patients were, on average, $76.5+/-8.7$ years old and had $13.2+/-3.8$ years of education. The median and modal Clinical Dementia Rating score was 2 (range, 1 to 4 ). A total of 35 participants (28\%) were residing in a nursing home, and 60 participants (48\%) were receiving a level of skilled nursing care comparable with a nursing home. A total of $61 \%$ of the cohort were women.

Figure 1 and the table present data from the initial visit at which both the TSI and the mMMSE were administered. The correlation between the TSI and the mMMSE was high $(r=0.82, p<0.0001)$. Patients generally obtained higher percent-correct scores on the TSI than the mMMSE. Of note, there was a range of scores on the TSI among participants scoring near 0 points on the MMMSE (see figure 1).

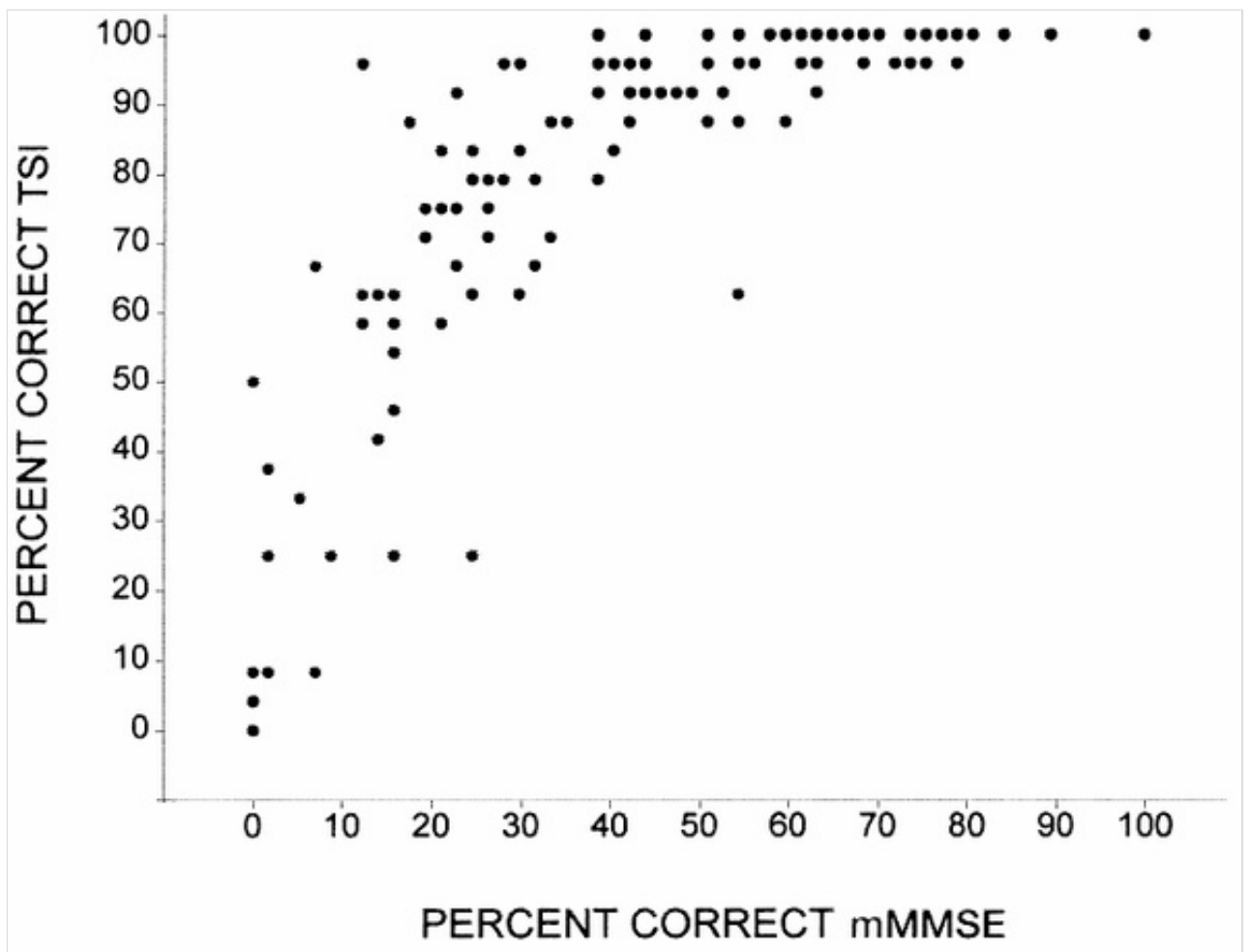

Figure 1. Percent correct ([score / maximum score] x 100) on the Test for Severe Impairment (TSI) and the modified Mini-Mental State Examination (mMMSE). 


\begin{tabular}{llc}
\hline & $\begin{array}{c}\text { TSI-mMMSE } \\
\text { Comparison, } \\
\mathrm{n}=125\end{array}$ & $\begin{array}{c}\text { TSI-MMSE } \\
\text { Comparison, } \\
\mathrm{n}=48\end{array}$ \\
\hline Mean (SD) TSI score & $17.6(7.9)$ & $16.3(8.4)$ \\
$\%$ Correct TSI score & 73.3 & 67.8 \\
Mean (SD) mMMSE score & $21.6(14.6)$ & $19.8(14.8)$ \\
$\%$ Correct mMMSE score & 38.0 & 34.8 \\
Mean (SD) MMSE score & N/A & $10.4(8.2)$ \\
$\%$ Correct MMSE score & N/A & 34.7 \\
\hline $\begin{array}{l}\text { TSI = Test for Severe Impairment; mMMSE = modified Mini- } \\
\text { Mental State Examination; MMSE }=\text { Mini-Mental State Exami- } \\
\text { nation; N/A = not available. }\end{array}$ &
\end{tabular}

Table Scores obtained by AD patients in the reported comparisons of the TSI with the mMMSE and MMSE at the time of the first administration of the TSI

A total of 66 participants had both TSI and mMMSE data for three or more visits and were included in the slope analyses to assess longitudinal rates of change of these measures. Duration of follow-up ranged from 1.5 to 4.5 years. The annual rate of change was $-9.5+/-1.0 \%$ on the mMMSE and $-18.0+/-1.8 \%$ on the TSI $(t[65]=5.7, p<0.0001)$. When analyses were restricted to those participants scoring less than 20 points on the mMMSE, which corresponds to a score of 10 points on the MMSE $(n=50)$, the annual rate of change was $-7.5+/-0.8 \%$ on the mMMSE and $-20.4+/-2.0 \%$ on the TSI $(t[49]=8.2, p<0.0001)$.

Cox regression analyses revealed that either the mMMSE or the TSI score contributed significantly to the prediction of time until death when included as the sole variable in the model. Specifically, a relatively lower score on either measure was associated with a significantly increased risk of death. When the two measures were included in the model simultaneously, however, only the TSI was a significant predictor (relative risk [RR], $0.94 ; 95 \% \mathrm{Cl}, 0.88$ to 0.99$)$.

TSI and MMSE. In addition to the TSI and the mMMSE, the MMSE was administered to 48 participants at the Johns Hopkins site, for a total of 206 visits. Figure 2 and the table present data from the initial visit at which all measures were administered. The correlation between the TSI and the MMSE was high $(r=0.83, p<0.0001)$. Again, there was a range of scores on the TSI among those obtaining very low scores on the MMSE (see figure 2). 


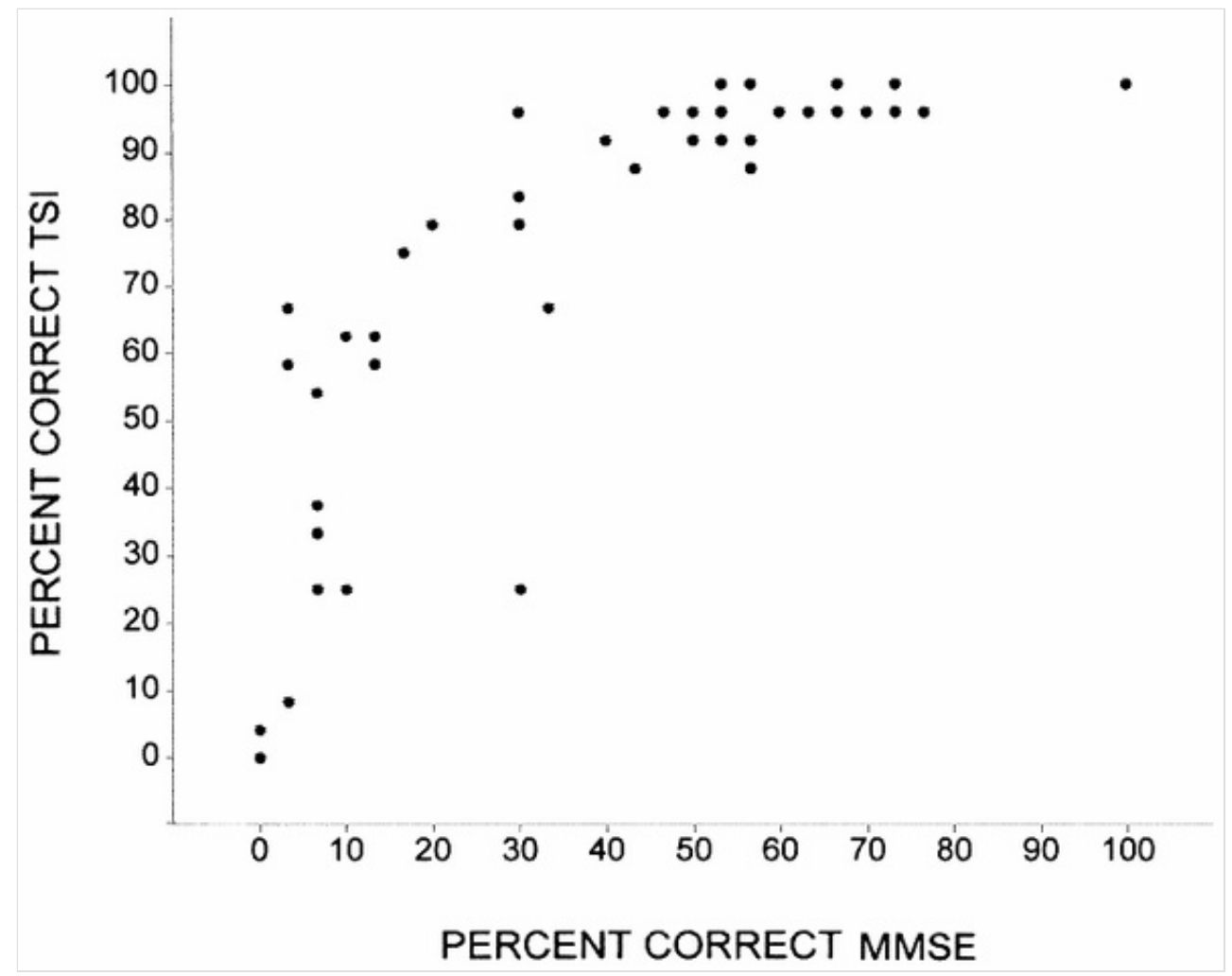

Figure 2. Percent correct ([score / maximum score] x 100) on the Test for Severe Impairment (TSI) and the 30-item Mini-Mental State Examination (MMSE).

A total of 33 participants had sufficient follow-up data on both the TSI and the MMSE to be included in the slope analyses to assess longitudinal rates of change. The rate of decline was $-8.4+/-1.9 \%$ per year on the MMSE and $-19.0+/-2.9 \%$ per year on the TSI $(t[32]=5.2, p<0.0001)$. When analyses were restricted to those scoring less than 10 points on the MMSE $(n=24)$, the annual rate of change was $-5.1+/-1.2 \%$ per year on the MMSE and $-20.5+/-3.4 \%$ per year on the TSI $(t[23]=5.9, p<0.0001)$.

The TSI was a significant predictor of survival until death (RR, $0.95 ; 95 \% \mathrm{Cl}, 0.91$ to 0.99$)$, whereas the MMSE was not.

Discussion. Commonly used mental status examinations may fail to detect relatively preserved abilities in patients with severe dementia. This lack of sensitivity is a limitation in both clinical practice and longitudinal research because it precludes the assessment of changes in cognition as dementia progresses. Our results confirm that the TSI is better able to assess cognitive function and to detect cognitive decline in severely demented patients with AD than the MMSE or the mMMSE.

The TSI was highly correlated with the MMSE and the MMMSE, but these measures were not redundant. Participants generally performed better on the TSI, particularly those scoring in the lower range of scores on the other instruments. Because floor effects were minimized on the TSI, there was a range of scores within which decline could be demonstrated. Results suggest that the utility of the TSI is greatest in patients who fail the majority of items on standard mental status examinations. Practitioners are likely to find the TSI most useful in patients scoring less than 10 to 15 points on the MMSE. The TSI extends the lower bound of the range of abilities that can be detected in severe dementia; but, like other mental status examinations, it was not useful in assessing patients who were nonresponsive.

There has been some speculation that the rate of cognitive decline slows in the severe stage of dementia.7 Results of the current study suggest that the inexorable cognitive decline that defines $A D$ continues into the severe stages of this disorder; however, the ability to detect this decline with standard mental status examinations is limited.

\section{References}

1. Albert M, Cohen C. The test for severe impairment: an instrument for the assessment of patients with severe cognitive dysfunction. J Am Geriatr Soc 1992;40:449-453. Bibliographic Links | [Context Link]

2. Saxton J, Swihart A, McGonigle-Gibson K, Miller V, Boller F. Assessment of the severely impaired patient: description and validation of a new neuropsychological test battery. Psychol Assess 1990;2:298-303. [Context Link]

3. Peavy GM, Salmon DP, Rice VA, et al. Neuropsychological assessment of severely demented elderly: the severe cognitive impairment profile. Arch Neurol 1996;53:367-372. Bibliographic Links | [Context Link]

4. Stern Y, Folstein M, Albert M, et al. Multicenter study of predictors of disease course in Alzheimer disease (the "Predictors Study"). I. Study design, cohort description, and intersite comparisons. Alzheimer Dis Assoc Disord 1994;7:3-21. [Context Link] 
5. Stern Y, Sano M, Paulson J, Mayeux R. Modified Mini-Mental State Examination: validity and reliability. Neurology 1987;37(suppl 1):179. Abstract. [Context Link]

6. Folstein MF, Folstein SE, McHugh PR. 'Mini-Mental State': a practical method for grading the cognitive state of patients for the clinician. $J$ Psychiatr Res 1975;12:189-198. Bibliographic Links | [Context Link]

7. Stern RG, Mohs RC, Davidson M, et al. A longitudinal study of Alzheimer's disease: measurement, rate, and predictors of cognitive deterioration. Am J Psychiatry 1994;151:390-396. Bibliographic Links |[Context Link]

\section{IMAGE GALLERY}

Select All

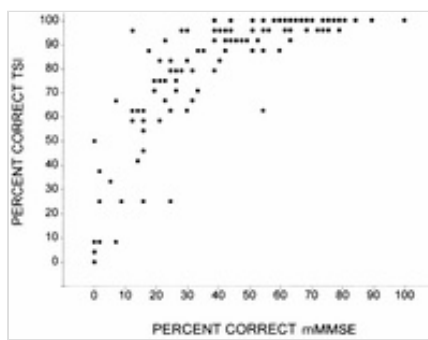

Figure 1

Back to Top
Export Selected to PowerPoint

\begin{tabular}{|c|c|c|}
\hline Parameter & $\begin{array}{l}\text { TS1-miBSSE, } \\
\text { Comparieno, } \\
=-125\end{array}$ & 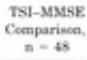 \\
\hline Yean (SD) TSI neepe & $17.6(8.9)$ & $163(8.4)$ \\
\hline \& Correst TSt sore & 733 & 67.8 \\
\hline Nean (SD) mMMSE nore & $21.6(146)$ & $19.8(14.8)$ \\
\hline 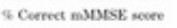 & 380 & 348 \\
\hline Mean (SD) MDSSE secere & $\mathrm{N} / \mathrm{A}$ & $10.4(8.2)$ \\
\hline SCerret MMSE nore & $\mathrm{N} / \mathrm{A}$ & 3. 7 \\
\hline
\end{tabular}

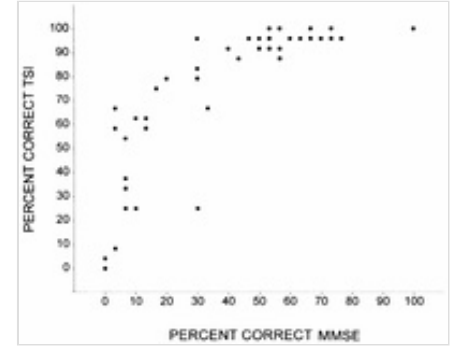

Figure 2

About Us Contact Us Privacy Policy Terms of Use 